\title{
Avaliação do uso de leitos de UTI de Covid-19 em Uberaba por meio da Teoria das \\ Filas
}

\section{Evaluation of the occupation of Covid-19 ICU beds in Uberaba according to the Queue \\ Theory}

\author{
Tarley Afonso da Silva ${ }^{1}$ \\ Universidade Federal do Triângulo Mineiro (UFTM), Uberaba, MG, Brasil \\ https://orcid.org/0000-0001-9004-6835, 0 http://lattes.cnpq.br/3121234466242664 \\ Michelli Maldonado 2 \\ Universidade Federal do Triângulo Mineiro (UFTM), Uberaba, MG, Brasil \\ https://orcid.org/0000-0002-8822-5508, , http://lattes.cnpq.br/9280860158879234
}

\begin{abstract}
Resumo: A aplicação de modelos da Teoria das Filas na área da saúde tem sido um solucionador de problemas sobre o congestionamento e dimensionamento de demanda no sistema hospitalar. $\mathrm{O}$ objetivo do trabalho foi desenvolver uma análise da ocupação dos leitos de Unidade de Terapia Intensiva, destinados a pacientes com Covid-19 durante o ano de 2020 na cidade de Uberaba, MG, por meio da Teoria das Filas. A pesquisa baseia-se em simulações da quantidade de pacientes que são internados por dia, de acordo com os dados oferecidos pela prefeitura de Uberaba, em relação à quantidade de leitos e à média de tempo que cada paciente fica internado. Assim, foi possível calcular quando o sistema hospitalar da cidade não suportaria a demanda e faltariam leitos para atender os cidadãos da cidade de Uberaba. Dos quatro cenários avaliados, apenas um apresentou um possível colapso no sistema de saúde.
\end{abstract}

Palavras-chave: Teoria das Filas; sistema hospitalar; leitos hospitalares; Covid-19.

\begin{abstract}
The application of Queue Theory models in the health area has been a problem solver about congestion and demand dimensioning in the hospital system. The objective of the work was to develop an analysis of bed occupancy in the Covid-19 Intensive Care Unit during the year 2020 in the city of Uberaba, MG. This research, that follows the Queue Theory, is based on simulations on the number of patients who are hospitalized per day, according to the data offered by the city of Uberaba in relation to the number of beds and the average time that each patient is hospitalized. Thus, it was possible to calculate when the city's hospital system would not support the demand and there would be a lack of beds to serve the citizens of the city of Uberaba. Out of the four scenarios presented, only one presented a possible collapse in the health system.
\end{abstract}

Keywords: Queuing Theory; hospital system; hospital beds; Covid-19.

Data de submissão: 16 de abril de 2021.

Data de aprovação: 3 de agosto de 2021.

\section{Introdução}

A pandemia provocada pela Covid-19 teve um avanço preocupante no ano de 2020 em vários países. A progressão do número de casos e as limitações existentes nos serviços de saúde disponíveis à população tornaram ainda mais crítico e complexo o seu enfrentamento. A doença causada pela infecção do novo coronavírus (SARS-CoV-2) foi considerada como uma pandemia

${ }^{1}$ Currículo sucinto: Licenciando em Matemática pela Universidade Federal do Triângulo Mineiro. Contribuição de autoria: Primeira Redação e Análise dos dados. Contato: d201720536@uftm.edu.br.

2 Currículo sucinto: Bacharel, mestre e doutora em Matemática pela Universidade Estadual Paulista (UNESP), professora do departamento de Matemática da Universidade Federal do Triângulo Mineiro. Contribuição de autoria: Supervisão e Escrita - Revisão e Edição. Contato: michelli.oliveira@uftm.edu.br. 
pela Organização Mundial de Saúde (OMS) no dia 11 de março de 2020 (WHO, 2020). Desde então, o mundo enfrenta o que já pode ser considerada a pior crise sanitária desde o surto da Influenza H1N1 em 1918.

No Brasil, o primeiro caso foi registrado no dia 25 de fevereiro de 2020 e o primeiro óbito ocorreu na cidade de São Paulo em 17 de março de 2020. No estado de Minas Gerais, o primeiro caso ocorreu dia 08 de março com uma moradora de Divinópolis, sendo o sétimo estado brasileiro a ter casos confirmados. Já na cidade de Uberaba, na região do Triângulo Mineiro, no dia 18 de março de 2020 foi registrado o primeiro caso, sendo a primeira morte 21 dias depois, em 08 de abril de 2020 (G1 TRIÂNGULO E ALTO PARANAÍBA, 2020).

As medidas de isolamento na cidade de Uberaba começaram dia 18 de março de 2020, com o Decreto no 5351 (UBERABA, 18 mar. 2020) que determinava suspender serviços de cirurgias, atendimentos em clínicas e demais áreas relacionadas com a saúde. Logo depois, as medidas de isolamento tomaram proporções maiores. Com o Decreto $n^{\circ} 5372$ (UBERABA, 20 mar. 2020) do dia 20 de março de 2020, foi determinado o fechamento dos estabelecimentos comerciais, locais de lazer e restaurantes, permanecendo abertos apenas serviços considerados essenciais, como supermercados, farmácias e hospitais. O fechamento foi determinado até a data de 30 de abril de 2020, porém, só com o Decreto n 5555 (UBERABA, 25 maio 2020) do dia 25 de maio de 2020, foi permitida a reabertura dos estabelecimentos com medidas de proteção obrigatórias, como distanciamento social, higienização e uso obrigatório de máscaras. Até o final do ano de 2020, o Decreto impedia o funcionamento das redes de ensino presencialmente, restaurantes, academias, festas e afins. No entanto, em junho houve uma liberação parcial de bares e de restaurantes, que foram autorizados, com várias restrições e sem a permissão da distribuição de bebidas. Na data de 31 de julho foi liberado o consumo de bebidas alcoólicas em bares e estabelecimentos, respeitando as medidas de prevenção e distanciamento, e também foi liberada a prática de exercícios físicos de forma individual, acarretando a reabertura de academias (UBERABA, 31 jul. 2020).

Almeida, Duarte-Filho e Nery (2020) destacam que uma das características mais marcantes do novo coronavírus é sua alta transmissibilidade, cujo aumento veloz no número de casos, especialmente de pacientes em estado grave, pode sobrecarregar, facilmente, o sistema de saúde de um país, levando-o ao colapso. Ainda segundo os autores, o cenário mais catastrófico do colapso do sistema de saúde é a saturação de leitos de Unidade de Terapia Intensiva (UTI), haja vista que, muitas vezes, os pacientes em estado mais crítico necessitam de internamento nestas unidades e da utilização de respiradores mecânicos artificiais, para que as suas chances de sobrevivência aumentem diante da síndrome respiratória aguda grave que pode ser desenvolvida quando uma pessoa contrai o vírus. 
No período de estudo, no ano de 2020, a cidade de Uberaba tinha 67 leitos de UTI destinados exclusivamente para pacientes com Covid-19 (UBERABA CONTRA COVID, 2020). Considerando que há um processo de chegada do paciente, um processo de atendimento durante a ocupação dos leitos, e uma liberação do leito com a alta ou o óbito, pode-se considerar o problema de possível saturação dos leitos como um problema da Teoria das Filas.

A aplicação de modelos da Teoria das Filas na área da saúde tem sido um solucionador de problemas sobre o congestionamento e dimensionamento de demanda no sistema hospitalar. A Teoria das Filas consiste de métodos analíticos que determinam e avaliam as medidas de desempenho relacionadas aos processos internos e externos, proporcionando indicadores que auxiliam as instituições hospitalares nas tomadas de decisões, para redução das probabilidades de filas e o tempo de espera dos pacientes (FOGLIATTI; MATTOS, 2007).

O objetivo deste trabalho é analisar a evolução da ocupação de leitos de UTI destinados para pacientes de Covid-19 na cidade de Uberaba-MG no ano de 2020 usando Teoria das Filas.

\section{Referencial Teórico}

A Teoria das Filas é aplicada em problemas que envolvem fluxos de serviços. De um lado existe uma entidade necessitando de serviço e, do outro lado, existe outra entidade fornecendo o serviço desejado. O primeiro passo é certificar-se de que a quantidade média de serviço ofertada seja capaz de atender a demanda média solicitada para que gargalos permanentes não ocorram. De acordo com Viana (2003), se a média da oferta de serviço não for pelo menos igual às demandas médias, um acúmulo de serviço irá ocorrer até que a demanda por serviço seja reduzida ou a oferta aumente. O estudo da Teoria das Filas tem como objetivo propor melhorias para o desempenho do sistema, ou seja, a melhor utilização dos recursos de serviços considerando o ponto de vista do cliente e do servidor. As medidas de desempenho mais importantes de um sistema, como o número médio de clientes no sistema e na fila, o comprimento médio da fila, o tempo médio de espera no sistema e na fila e a taxa média de ocupação do serviço, auxiliam na tomada de decisão para minimizar o tamanho das filas e a permanência de clientes no sistema (COSTA, 2006).

A primeira aplicação da Teoria das Filas se deu no início do século XX com o matemático dinamarquês Agner Krarup Erlang (1878) (LONGARAY et al., 2016), para resolver um problema das redes telefônicas. Além de companhias telefônicas, tem-se aplicações dessa teoria em muitas outras áreas. Exemplos disso é a distribuição de mercadorias, em que pode-se ter um estudo melhorando a dinâmica da empresa, o local em que um depósito é construído a fim de maximizar o desempenho, além também do gerenciamento dos depósitos em que se analisa onde cada material/produto deve ficar para um melhor funcionamento, no aeroporto em que os aviões precisam esperar para fazer a decolagem analisando o tempo de espera, a fila gerada e 
quantidade necessária de pista de pouso. Tem também a otimização em filas comuns como filas de banco, supermercado, farmácia, lotéricas e todas as demais. Existem inúmeras situações em que pode ser aplicada a Teoria das Filas e, para o estudo deste artigo, foi utilizada a Teoria das Filas no caso hospitalar, em que se analisa o tempo que o paciente esperará para ser atendido pelo sistema e se esse sistema está conseguindo atender de uma forma positiva a população demandante (MAGALHÃES, 1996).

Uma fila é a representação de uma linha de pessoas, veículos, objetos que aguardam sua vez para serem atendidos. Segundo a definição dos pesquisadores Fogliatti e Mattos (2007), um "Sistema com Fila" se baseia em qualquer processo onde usuários oriundos de uma determinada população chegam para receber um serviço pelo qual esperam, saindo do sistema assim que o serviço é completado.

A formação de filas ocorre se a demanda de pessoas ultrapassa a capacidade do sistema de fornecer serviços em certo tempo. Assim, a Teoria das Filas auxilia no projeto e na operação dos sistemas para encontrar um equilíbrio adequado entre os custos de oferecer serviços no sistema (por exemplo, custo operacional, custo de capacidade) e os custos dos atrasos sofridos pelos usuários das filas (ARENALES et al., 2006).

De acordo com Mendonça (2014), um sistema com fila é composto por usuários, postos de atendimento e por um espaço designado para a espera. Assim, os usuários chegam em um determinado tempo, caracterizando o processo de chegada $(\lambda)$. Eles são atendidos em determinado canal ou postos de atendimento; se os postos estão todos ocupados os usuários aguardam em uma fila. Assim que um canal ou posto de serviço fica livre, um dos usuários da fila é chamado para atendimento e, uma vez completado o serviço, o usuário é liberado do sistema. Logo, é possível determinar o processo de atendimento com o número de usuários atendidos em um espaço durante um certo período de tempo, $(\mu)$. A Figura 1 representa um modelo básico de filas. Existe o conjunto da população, e alguns elementos deste conjunto podem se transformar em clientes ao entrar no sistema. Dependendo da quantidade de clientes, pode-se ter uma fila de espera e cada cliente é atendido por um servidor. O número de servidores depende do contexto estudado. Ao finalizar o atendimento, o cliente deixa de fazer parte do sistema.

Arenales et al. (2006) destacam que é importante determinar a estrutura do sistema, que pode ser classificada em: fila única e único servidor, fila única e múltiplos servidores em paralelo, múltiplas filas e múltiplos servidores em paralelo, fila única e múltiplos servidores em série.

A disciplina da fila é a regra que define qual o próximo cliente a ser atendido, ou seja, a ordem em que os usuários são selecionados da fila para o atendimento (CHWIF; MEDINA, 2014). 
Figura 1 - Representação gráfica do funcionamento de um Sistema de Filas

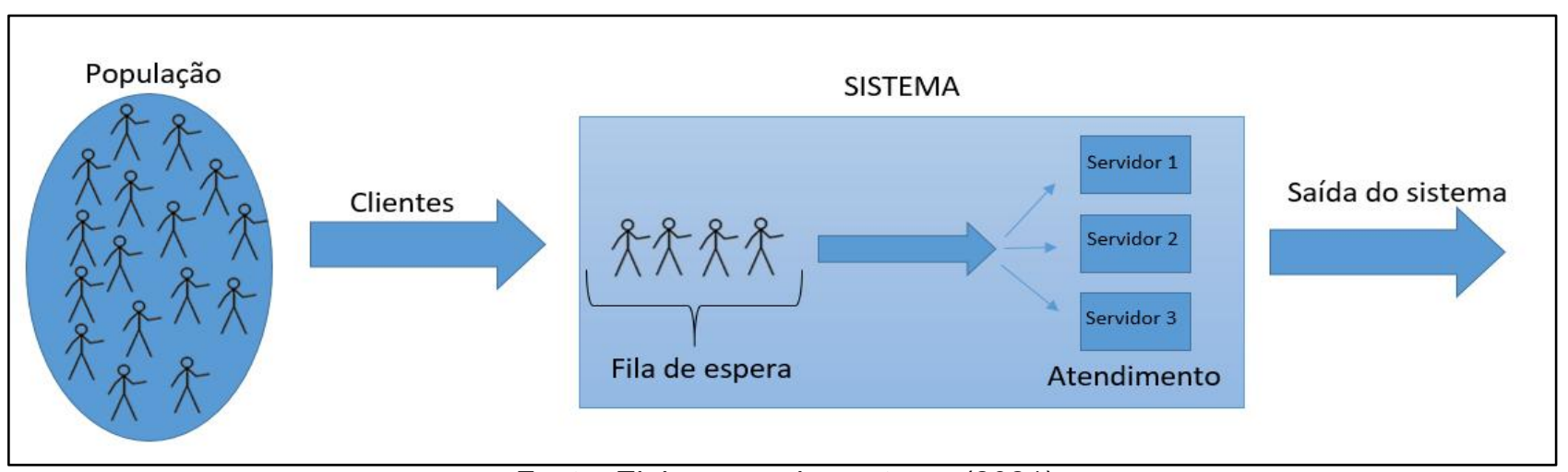

Fonte: Elaboração dos autores (2021).

Arenales et al. (2006) falam que todas essas variações de sistemas de filas levam a uma variedade de modelo de filas e, para simplificar essa análise, os mais simples são classificados conforme a notação de Kendall-Lee:

\section{$\mathrm{A} / \mathrm{B} / \mathrm{s} / \mathrm{C} / \mathrm{K} / \mathrm{N}$}

onde,

A - Processo de chegada

B - Processo de atendimento

s - Número de atendentes (em paralelo)

C - Número máximo de clientes no sistema (capacidade do sistema)

$\mathrm{K}$ - Tamanho da população

$\mathrm{N}$ - Regra da fila (disciplina de atendimento)

O modelo conhecido como Markoviano/Markovian/Single (modelo $\mathrm{M} / \mathrm{M} / \mathrm{s}$ ) é clássico e o mais simples da literatura. Esse modelo recebe esse nome porque assume uma distribuição Markoviana dos intervalos de chegada, que são distribuídos de acordo com uma distribuição exponencial independente e identicamente distribuída (HILLIER; LIEBERMAN, 2012).

O modelo $\mathrm{M} / \mathrm{M} / \mathrm{s}$ é baseado na interação entre dois parâmetros: a) a taxa média de chegada, $\lambda$; b) a taxa média de serviço, $\mu$. Para garantir a estabilidade do sistema, a taxa total de serviços, deve exceder a taxa de demanda $(\lambda<s \mu)$. Caso contrário, o sistema torna-se instável e a fila tende ao infinito.

Uma vez definidos os parâmetros de entrada e de saída do sistema de filas, é possível determinar, para diferentes cenários, a taxa de ocupação, o número médio de clientes na fila, o número médio de clientes no sistema e a probabilidade de $n$ clientes estarem no sistema. As equações (1)-(8) são as fórmulas do modelo M/M/s baseado em Arenales et al. (2006). 
Na Equação (1), a notação $r$ representa a razão entre a taxa de chegada e a taxa de atendimento.

$$
r=\frac{\lambda}{\mu}
$$

A taxa de ocupação $\rho$, apresentada na Equação (2), equivale ao valor da razão entre a taxa de chegada de usuários no sistema e a taxa total disponível de serviço no sistema. Ela traz valores maiores ou iguais a zero e geralmente menores que 1. A partir do momento que a taxa ultrapassa o valor de 1, significa que a demanda média excede a capacidade média do sistema de oferecer serviços, fazendo com que a fila aumente indefinidamente com o tempo.

$$
\rho=\frac{\lambda}{s \mu}
$$

A taxa de desocupação $P_{0}$ é a probabilidade de o sistema estar vazio, ou seja, a probabilidade do servidor estar desocupado, e é dada pela Equação (3). Só é possível calcular a taxa de desocupação se $\rho<1$, pois essa é a condição em que o sistema M/M/s atinge o equilíbrio.

$$
P_{0}=\sum_{n=0}^{S-1}\left[\frac{r^{n}}{n !}+\frac{r^{s}}{s !}\left(\frac{1}{1-\rho}\right)\right]
$$

O número médio de clientes na fila $\left(L_{q}\right)$, dado pela Equação (4), representa a média da quantidade de clientes que estão na fila esperando para serem atendidos, ou seja, o comprimento da fila. O número médio de clientes no sistema ( $L$ ), dado pela Equação (5), é a quantidade de pessoas que se encontram no sistema, ou seja, a média de pessoas que estão esperando na fila mais as pessoas que estão em atendimento.

$$
\begin{gathered}
L_{q}=\frac{P_{0} r^{s} \rho}{s !(1-\rho)^{2}} \\
L=L_{q}+r
\end{gathered}
$$

O tempo médio gasto de espera na fila por cliente $\left(W_{q}\right)$, dado pela Equação (6), é a média do tempo que cada cliente gasta esperando na fila para ser atendido.

$$
W_{q}=\frac{L_{q}}{\lambda}
$$

O tempo médio de espera no sistema por cliente ( $W$ ), dado pela Equação (7), é a média da soma do tempo em que os clientes gastam na fila com o tempo para ser atendido; ou seja, todo tempo que o cliente gasta dentro do sistema. 


$$
W=W_{q}+\frac{1}{\mu}
$$

Na Equação $8, P_{n}$ é a probabilidade de um certo número $n$ clientes estarem no sistema ( $n$ igual a um valor escolhido).

$$
P_{n}=\left\{\begin{array}{l}
\frac{r^{n}}{s ! s^{(n-s)}} P_{0}, \text { se } n \geq s \\
\frac{r^{n}}{n !} P_{0}, \text { se } 0 \leq n \leq s
\end{array}\right.
$$

De acordo com Yaduvanshi, Sharma e More (2019), existem muitas pesquisas sobre análise de filas no contexto hospitalar. Os autores apresentaram uma análise usando a Teoria das Filas em um movimentado hospital da Índia. Segundo Mathias, Coelho e Gonçalves (2015, p. 4),

Os estudos de filas em instituições hospitalares possuem características próprias em suas operações de alta complexidade. Outro fator considerável é o paciente, visto como participante ativo e determinante quanto aos processos dos serviços de internação. Isto se dá por sua cooperação e evolução clínica, alterando significativamente no desempenho das prestações de serviços da saúde.

Marinho (2006) estuda as filas para transplantes no Sistema Único de Saúde, estabelecendo um quadro geral da situação deste problema no Brasil, com o auxílio do modelo M/M/s da Teoria das Filas. Goldwasser et al. (2016) usam a Teoria das Filas para determinar o número necessário de leitos públicos de unidades de terapia intensiva para adultos do estado do Rio de Janeiro para atender a demanda existente. Os resultados mostram que é necessário um incremento de $122 \%$ dos leitos para garantir a estabilidade do sistema, e de $134 \%$ para um tempo máximo de espera de seis horas.

Marinho (2020) apresenta um estudo, usando Teoria das Filas, para analisar vantagens e desvantagens da eventual adoção de uma fila única em unidade de terapia intensiva em todos os hospitais públicos e privados brasileiros, no contexto da pandemia da Covid-19. A análise utiliza elementos teóricos, e de resultados práticos, observados em economia da saúde e, de modo complementar, em Teoria das Filas. Essa análise foi em função da gravidade da situação atual e da possibilidade de que a fila única seja adotada na presente pandemia, ou que venha a ser novamente objeto de debate, em eventuais futuras pandemias, ou em epidemias que ocorram no país.

\section{Metodologia}

O estudo é de natureza quantitativa, observacional com geração de cenários. Considera-se um sistema de fila $\mathrm{M} / \mathrm{M} / \mathrm{s}$ para analisar o uso dos leitos de unidade de terapia intensiva da cidade de Uberaba destinados a pacientes com Covid-19. O parâmetro foi considerado como o número de leitos disponíveis no momento do estudo, 67. A taxa média de serviço, $\mu$, foi considerada de 
18 dias, dado disponibilizado pela Secretaria Municipal de Saúde de Uberaba nos boletins diários (UBERABA CONTRA COVID, 2020).

Para a taxa média de chegada, $\lambda$, a fins de análise, são atribuídos diversos valores para a geração de diferentes cenários. Posteriormente, os cenários são analisados e discutidos.

A proposta foi projetar cenários e estabelecer sob quais condições pode-se ter formação de filas, ou seja, saturação dos leitos. Os cenários foram desenhados considerando diversas taxas médias de chegadas por dia. Assim, foi possível calcular a taxa de ocupação, a probabilidade de saturação dos leitos e apresentar a evolução do uso dos leitos com o passar dos dias. Inicialmente todos os leitos foram considerados desocupados. Cada cenário considera uma taxa média de pessoas chegando ao hospital para internação por dia, sendo analisadas quatro situações: $\lambda=1, \lambda=2, \lambda=3$ e $\lambda=4$ pacientes/dia.

Até a finalização do presente trabalho, o sistema de saúde da cidade de Uberaba encontrava-se em situação confortável no atendimento dos pacientes com Covid-19 e sem indicativos de colapso. Ao simular os valores para o parâmetro $\lambda$, percebeu-se que situações inferiores a 4 pacientes/dia se aproximava da realidade da cidade, enquanto $\lambda \geq 4$ pacientes/dia indicava um possível descontrole no atendimento. Sendo assim, foi escolhido $\lambda=1, \lambda=2$ e $\lambda=3$ pacientes/dia por se aproximar da situação real e $\lambda=4$ pacientes/dia para simular 0 primeiro cenário de colapso e comparar com os demais.

Todos os dados, cálculos e tabelas foram tabulados e gerados com o auxílio do Microsoft Excel.

\section{Resultados e Discussões}

A Tabela 1 apresenta os resultados das principais medidas de desempenho de Teoria das Filas de um modelo M/M/s. Por se considerar na construção dos cenários a entrada de novos pacientes diariamente é de se esperar que a probabilidade de desocupação do sistema seja praticamente nula, por isso, seu valor não é explícito na Tabela 1.

A taxa de chegada é fixada em cada cenário e a Tabela 1 apresenta a taxa de ocupação para o equilíbrio do sistema em cada cenário. A saturação dos leitos é caracterizada pela ocupação de $100 \%$ deles, ou seja, 67 leitos, e apresenta-se a probabilidade de isso acontecer em cada cenário. Um ponto de alerta na cidade é definido quando $70 \%$ dos leitos estão ocupados, nesse exemplo, 47 deles; nesta situação os gestores públicos estariam em busca de soluções de mitigação de casos. Por fim, a Tabela 1 apresenta o número médio de usuários no sistema em equilíbrio. 
Tabela 1 - Medidas de desempenho do modelo no contexto do uso de leitos de UTI de Covid-19 em Uberaba

\begin{tabular}{|c|c|c|c|c|}
\hline Cenários & $\mathbf{1}$ & $\mathbf{2}$ & $\mathbf{3}$ & $\mathbf{4}$ \\
\hline Taxa de Chegada & 1 & 2 & 3 & 4 \\
\hline Taxa de Ocupação & $26,87 \%$ & $53,73 \%$ & $80,60 \%$ & $107,46 \%$ \\
\hline Probabilidade de 67 pessoas no Sistema (100\%) & $0,00 \%$ & $0,00 \%$ & $1,13 \%$ & $0 \%$ \\
\hline Probabilidade de 47 pessoas no Sistema (70\%) & $0,00 \%$ & $1,26 \%$ & $3,58 \%$ & $0 \%$ \\
\hline Número médio de usuário no sistema & 18 & 35 & 54 & 46 \\
\hline
\end{tabular}

Fonte: Elaboração dos autores (2021).

A Tabela 1 contém os resultados dos cálculos realizados com as Equações (1)-(8). No Cenário 1, consideramos uma pessoa chegando por dia, ou seja, $\lambda=1$. Usamos a Equação (2), $\rho=\frac{\lambda}{s \mu}$, para encontrar a taxa de ocupação, lembrando que $\mu$ vale $\frac{1}{18}$ visto que o paciente fica internado por 18 dias e o valor de $s$ é igual a 67, que são os leitos disponíveis.

Para determinar as probabilidades usamos a Equação (8) considerando $n \geq 67$, $P_{n}=\frac{r^{n}}{s ! s^{(n-s)}} P_{0}$ no caso de $n=67$ o sistema ficaria $100 \%$ ocupado e para $n=47$ o sistema ficaria com $70 \%$ de ocupação.

E, por fim, usamos a Equação (5), $L=L_{q}+r$, para calcular a quantidade de usuários dentro do sistema. Após isso, repetimos todo o processo para $\lambda=2, \lambda=3$ e $\lambda=4$.

O Gráfico 1 apresenta a taxa de ocupação ao longo de um período de 30 dias para cada um dos cenários propostos. Os três primeiros cenários atingem o equilíbrio sem saturar. $\mathrm{O}$ Cenário 4 já apresenta saturação dos leitos após 18 dias.

Para o Cenário 1, pode-se perceber que a taxa de ocupação quando atinge a estabilidade do sistema, é dado por $26,87 \%$, mostrando que enquanto tiver apenas uma pessoa por dia entrando para ser internada nos leitos hospitalares, se tem o equilíbrio e ele não terá nenhuma saturação, visto que menos que $30 \%$ dos leitos serão ocupados nesse cenário.

Outros valores ínfimos que podemos considerar nesse primeiro cenário são o número médio de pacientes na fila $\left(L_{q}\right)$ e o tempo médio de espera dos clientes na fila $\left(W_{q}\right)$ visto que não teremos fila para utilizar o sistema. O tempo médio de espera dos clientes no sistema ( $W$ ) é 
aproximadamente 18 dias, que é exatamente o tempo médio que os pacientes ficam internados para recuperação dos efeitos da Covid-19.

Gráfico 1 - Taxa de ocupação ao longo de um período de 30 dias para os quatro cenários propostos

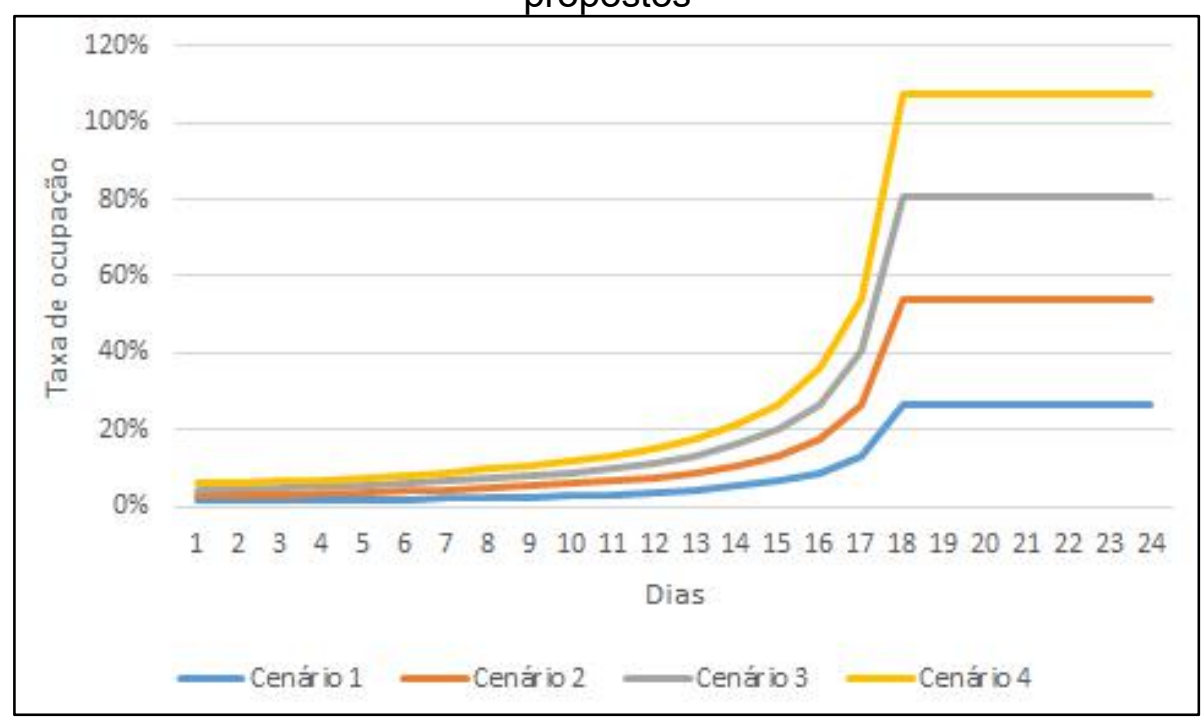

Fonte: Elaboração dos autores (2021).

O número médio de pacientes no sistema, ao decorrer do tempo, será de aproximadamente 18 pacientes por dia, levando em conta que se entra um paciente por dia e ficam internados em média por 18 dias; ao atingir o décimo oitavo dia, o hospital atingirá os 18 pacientes e, a partir disso, entrará um e sairá uma pessoa.

No Cenário 2, a taxa de pacientes que entram para serem internados por dia é igual a 2 pessoas/dia. Na Tabela 1 pode-se perceber que a taxa de ocupação atinge a estabilidade do sistema em $53,73 \%$, mostrando que enquanto tiver duas pessoas por dia entrando para serem internadas nos leitos hospitalares, se tem o equilíbrio e ele não terá saturação, visto que menos de $55 \%$ dos leitos serão ocupados nesse cenário.

Os valores do número médio de pacientes na fila $\left(L_{q}\right)$ e o tempo médio de espera dos clientes na fila $\left(W_{q}\right)$, apesar de terem aumentado, ainda são muito pequenos pois ainda não há fila para utilizar o sistema, tendo como (número médio de pacientes na fila e tempo médio de espera de clientes na fila). O tempo médio de clientes do sistema ( $W$ ) não muda nos cenários, mantendo os 18 dias, pois é exatamente o tempo médio que os pacientes ficam internados.

Já o número médio de pacientes no sistema aumenta para 36 pacientes por dia, pois levase em conta que, entrando dois pacientes por dia e ficando internados em média por 18 dias, ao atingir o décimo oitavo dia, o hospital atingirá os 36 pacientes e, a partir disso, atingirá o equilíbrio. 
A probabilidade de atingir os 67 leitos ocupados, apesar de ter aumentado um pouco o número de pacientes internados, ainda é inferior à quantidade de leitos oferecidos, assim, é bem improvável que atinja os 67 leitos ocupados nessas condições.

Para o Cenário 3, na Tabela 1 pode-se perceber que a taxa de ocupação quando o sistema atinge a estabilidade é dada por $80,60 \%$, mostrando que enquanto tiver três pessoas por dia entrando para serem internadas nos leitos hospitalares, tem-se o equilíbrio e não terá saturação. Mas também, pode-se perceber o aumento dos leitos ocupados, passando de $80 \%$, sendo considerado bem perto da ocupação total.

Os valores do número médio de pacientes na fila $\left(L_{q}\right)$ e o tempo médio de espera dos clientes na fila $\left(W_{q}\right)$, apesar de terem aumentado significativamente de valor, ainda são pequenos pois ainda não teremos fila para utilizar o sistema, tendo como resultados dos valores de $L_{q}$ e $W_{q}$ iguais a zero. O tempo médio de clientes do sistema $(W)$ mantém-se também em 18 dias.

Já o número médio de pacientes no sistema aumenta para 54 pacientes por dia, pois levase em conta que se entrarem três paciente por dia e ficarem internados em média por 18 dias, ao atingir o décimo oitavo dia, o hospital atingirá os 54 pacientes e, a partir disso, chegará ao equilíbrio. A probabilidade de atingir os 67 leitos ocupados, nesse cenário, aumentou significativamente. Antes era notável que a taxa era muito baixa, sendo desconsiderada, e agora ela começa a crescer aos poucos e atinge $1 \%$ de probabilidade de todos os leitos serem ocupados. Isso acontece porque houve um aumento das pessoas entrando, e caso tenha algum imprevisto durante o processo, há pequena chance de acontecer a lotação no sistema.

No Cenário 4, na Tabela 1, pode-se perceber que a taxa de ocupação quando o sistema atinge a estabilidade vale $107,46 \%$, mostrando que, enquanto tiver quatro pessoas por dia entrando para serem internadas nos leitos hospitalares, terá saturação no sistema. Assim, nessas condições, chegará em uma lotação, entrando em colapso. Como já visto, o tempo médio de clientes do sistema $(W)$ mantém-se em 18 dias, pois é exatamente o tempo médio que os pacientes ficam internados.

As demais taxas, por termos uma taxa de ocupação do sistema maior que $100 \%$, não são possíveis de se calcular. O sistema só funciona quando essa taxa é menor que $100 \%$, porque ele já prevê que nessas condições teria uma saturação, dando resultados negativos ao calcular as outras variáveis.

Nesse caso, teríamos a taxa de desocupação igual a zero, porque o sistema sempre estaria ocupado, teríamos filas para atendimento que não seriam supridas, o número médio de pacientes do sistema sempre seria o máximo (67 pacientes) a partir do décimo sétimo dia, e a 
probabilidade do sistema estar com os 67 leitos ocupados seria de $100 \%$, pois iria atingir esse valor também no décimo sétimo dia.

No Gráfico 2 pode-se analisar que todos os cenários vão crescendo gradativamente até chegar em um ponto de estabilidade, ou seja, o número de pacientes dentro do sistema cresce até atingir um equilíbrio, e depois disso se mantém na mesma quantidade de pessoas dentro deste.

Gráfico 2 - Número de dias necessários para cada cenário proposto atingir as taxas de ocupação de $30 \%, 50 \%, 70 \%$ e $100 \%$

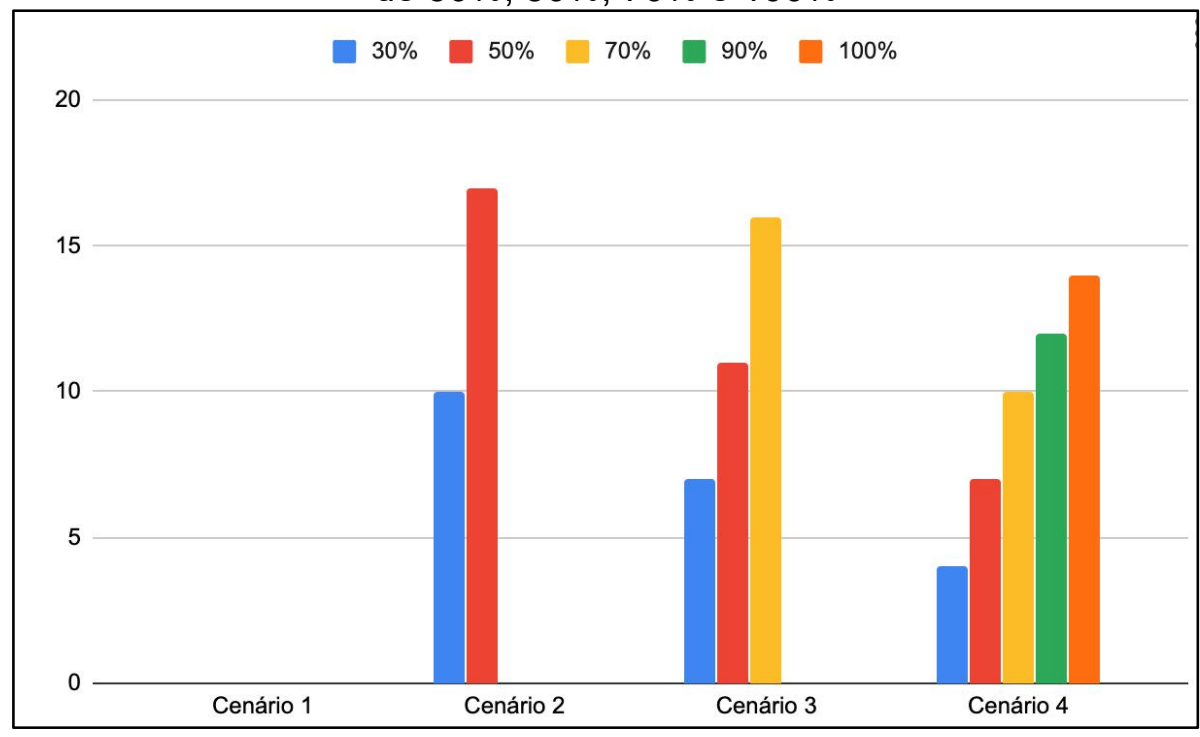

Fonte: Elaboração dos autores (2021).

Também é perceptível que a taxa de ocupação, até a o Cenário 3, está abaixo de 1, que representa $100 \%$. Isso significa que até tal cenário, é possível manter o sistema funcionando normalmente. A partir dele, no Cenário 4, observa-se que ele já ultrapassa, um pouco antes do décimo oitavo dia, os $100 \%$, mostrando que as condições de atendimento precisam ser mudadas e que haveria um possível problema na manutenção do sistema para funcionar em condições ideais. Neste caso, seria necessária a abertura de 5 novos leitos para o sistema não ultrapassar os $100 \%$. Ou então, abrir 20 novos leitos para o sistema trabalhar por volta de $80 \%$ de ocupação. A instalação de novos leitos gerará um custo, porém pode prevenir a perda de vidas.

No presente artigo as taxas de chegada foram fixadas e posteriormente calculadas as taxas de ocupação, mas é possível um cálculo reverso. Considerando os 67 leitos disponíveis, é possível estimar a taxa média de chegada para que o sistema não ultrapasse os $100 \%$. No contexto de Uberaba, $\lambda=3,7$, garante que o sistema não entre em colapso. Isso significa que pode variar o número de pessoas que são internadas por dia, mas em média $\lambda=3,7$. Ao pensar dessa forma, o gestor pode acompanhar a evolução das internações e decidir em quais os momentos medidas mais rígidas são necessárias. 
Considerando os dados disponibilizados no Boletim Diário da Prefeitura Municipal de Uberaba (UBERABA CONTRA COVID, 2020) e pelo Observatório Covid-19 Uberaba (2020), a média da taxa de ocupação dos leitos de UTI em 2020 foi de 43,05\%. O Gráfico 3 apresenta a evolução da ocupação dos leitos de UTI por semanas epidemiológicas durante o ano de 2020 referente à pandemia da Covid-19.

Gráfico 3 - Evolução da Taxa de Ocupação dos Leitos de UTI durante o período da pandemia no ano de 2020 (de junho a dezembro)

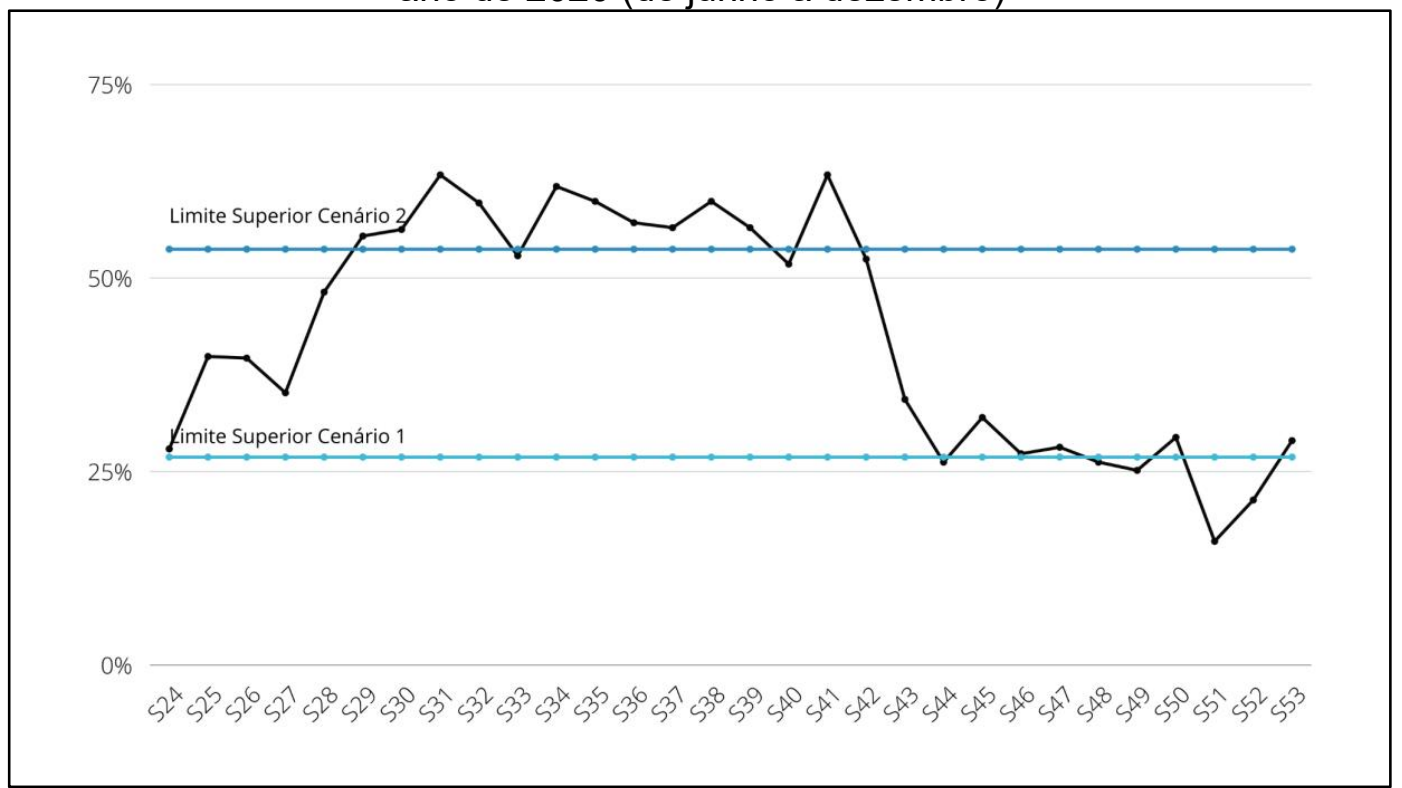

Fonte: Dados do Observatório Covid-19 Uberaba (2020).

Observa-se que a cidade de Uberaba se manteve no ano de 2020, entre a semana 24 a semana 53 (junho a dezembro), intercalando pelo Cenário 1 e pelo Cenário 2, com alguns momentos de mais alta, principalmente durante o pico da primeira onda da cidade que foi em setembro de 2020. Adicionalmente, cabe ressaltar que os modelos de Teoria das Filas mais simples são mais adaptáveis a situações emergenciais, em que a chegada de pacientes e as efetivas internações têm caráter mais aleatório do que em internações eletivas, muito mais sujeitas às interferências da gestão hospitalar.

\section{Conclusão}

Foi analisada a situação da cidade de Uberaba em relação aos leitos disponibilizados para o tratamento de pessoas infectadas pela Covid 19. Assim, os resultados estimam que com a taxa de chegada de pacientes por dia é possível que o sistema hospitalar seja capaz de atender as demandas da cidade sem que alguém seja prejudicado pela falta do atendimento. De acordo com os dados coletados, até o Cenário 3 as condições são favoráveis para o funcionamento do sistema, entrando em colapso no Cenário 4, em que o sistema teria uma superlotação não 
suportando a demanda prevista. Assim, se houver 4 pessoas por dia para serem internadas, em um dado momento, os leitos estarão totalmente ocupados, trazendo um déficit nos atendimentos, tendo as autoridades que entrar com outras medidas para controlar a situação ou então abrir no mínimo 5 novos leitos para atender a demanda.

Ao realizar o estudo, a intenção era exatamente prever quando o sistema entraria em colapso para analisar até em que cenário seria possível atender a todos da cidade de Uberaba. Com isso vimos que se a situação da cidade estiver nas condições propostas no Cenário 4 , o sistema hospitalar não suportaria a demanda, não conseguindo assim atender a todos que necessitam de cuidados médicos para sua sobrevivência, aumentando os casos de mortes, e de pessoas sem atendimentos.

Por fim, a intenção é alertar para que se tenham medidas de prevenção e isolamento para que não se chegue na situação da entrada de 4 pessoas por dia nos hospitais de Uberaba a serem internadas em decorrência da Covid-19, ou ainda, se houver tamanha demanda, alertar para a necessidade do aumento do número de leitos oferecidos à população uberabense.

\section{Referências}

ALMEIDA, F. A. G. de; DUARTE-FILHO, G. C.; NERY, M. B. M. Estatística da COVID-19 em Sergipe. Nota técnica, n. 1, 15 maio 2020. Universidade Federal de Sergipe. Disponível em: https://dfi.ufs.br/uploads/page attach/path/8398/NT01-SE-Francisco-Gerson-Matheus.pdf. Acesso em: 23 nov. 2021.

ARENALES, M.; ARMENTANO, V.; MORABITO, R.; YANASSE, H. Pesquisa Operacional. Rio de Janeiro: Elsevier, 2006.

CHWIF, L.; MEDINA, A. C. Modelagem e Simulação de Eventos Discretos: Teoria \& Aplicações. 4. ed. Rio de Janeiro: Elsevier, 2014.

COSTA, L.C. Teoria das Filas. Notas de Aulas. 2006. Universidade Federal do Maranhão UFMA, 2006. Disponível em: http://www.deinf.ufma.br/ mario/grad/filas/TeoriaFilas Cajado.pdf. Acesso em: 3 ago. 2021.

FOGLIATTI, M. C.; MATTOS, N. M. C. Teoria de Filas. Rio de Janeiro: Editora Interciência, 2007.

G1 TRIÂNGULO E ALTO PARANAÍBA. Prefeitura confirma primeira morte por coronavírus em Uberaba. G1 Triângulo e Alto Paranaíba, 10 abr. 2020. Disponível em:

https://g1.globo.com/mg/triangulo-mineiro/noticia/2020/04/10/prefeitura-confirma-primeira-mortepor-coronavirus-em-uberaba.ghtml. Acesso em: 31 maio 2021.

GOLDWASSER, Rosane Sonia; LOBO, Maria Stella de Castro; ARRUDA, Edilson Fernandes de; ANGELO, Simone Aldrey; LAPA E SILVA, José Roberto; SALLES, André Assis de; DAVID, Cid Marcos. Difficulties in access and estimates of public beds in intensive care units in the state of Rio de Janeiro. Revista de Saúde Pública, Rio de Janeiro, v. 50, 2016. DOI: https://doi.org/10.1590/S1518-8787.2016050005997. 
HILLIER, F. S., LIEBERMAN, G. J. Introdução à Pesquisa Operacional. 9. ed. São Paulo: McGraw-Hill, 2012.

LONGARAY, A. A., SANTOS, A. C., MUNHOZ, P. R.,CASTELLI, T. M. Análise da disciplina de filas no atendimento ao cidadão em agências da previdência social: um estudo de caso. Revista de Gestão em Sistemas de Saúde, v. 5, n. 1, p. 105-119, jan./jun. 2016. DOI: http://dx.doi.org/10.5585/rgss.v5i1.166.

MAGALHÃES, M. N. Introdução à Rede de Filas. São Paulo: Universidade de São Paulo, 1996, 127 p. Disponível em: https://www.ime.usp.br/ marcos/LivroSinape96Filas.pdf. Acesso em: 3 ago. 2021.

MARINHO, A. A proposta de adoção de fila única nas unidades de terapia intensiva e nas demais internações hospitalares, durante a pandemia de Covid-19 no Brasil: considerações teóricas do campo da economia da saúde sobre as alternativas disponíveis. Nota Técnica: Diretoria de Estudos e Políticas Setoriais de Inovação e Infraestrutura, maio de 2020. Brasília: Instituto de Pesquisa Econômica Aplicada. 28 p. Disponível em:

http://repositorio.ipea.gov.br/bitstream/11058/10011/1/NT 65 Diset \%20Proposta\%20de\%20Adoc ao\%20de\%20Fila\%20unica\%20nas\%20Unidades\%20de\%20Terapia\%20Intensiva.pdf. Acesso em: 31 maio 2021.

MARINHO, A. Um estudo sobre as filas para transplantes no Sistema Único de Saúde Brasileiro. Cadernos de Saúde Pública, v. 22, n. 10, p. 2229-2239, out. 2006. DOI: https://doi.org/10.1590/S0102-311X2006001000029.

MATHIAS, R. V.; COELHO, A. S.; GONÇALVES, M. B. Desempenho dos serviços de internações hospitalares utilizando o modelo de filas: estudo de caso em hospital de Santa Catarina. In:

SIMPÓSIO DE EXCELÊNCIA EM GESTÃO E TECNOLOGIA, 12., 2015, Resende, RJ. Anais [...]. Resende: AEDB, 2015. p. 1-12. Disponível em:

https://www.aedb.br/seget/arquivos/artigos15/34322379.pdf. Acesso em: 31 maio 2021.

MENDONÇA, Ednário Barbosa de. Teoria de Filas Markovianas e Aplicações. Orientadora: Divanilda Maia Esteves. 2014. 63 f. Trabalho de Conclusão de Curso (Graduação em Estatística) - Centro de Ciências e Tecnologia, Universidade Estadual da Paraíba, Campina Grande, 2014. Disponível em: http://dspace.bc.uepb.edu.br/jspui/handle/123456789/5265. Acesso em: 31 maio 2021.

OBSERVATÓRIO COVID-19 UBERABA. Uberaba, MG. 2020. Disponível em: https://coviduberaba.github.io/index.html\#uberaba. Acesso em: 31 maio 2021.

UBERABA. Decreto $\mathbf{n}^{\circ} \mathbf{5 3 5 1}$, de 18 de março de 2020. Impõe medidas emergenciais, decorrentes da propagação do Coronavírus - COVID-19 na Secretaria Municipal de Saúde do Município de Uberaba, Estado de Minas Gerais e dá outras providências. Disponível em: http://www.uberaba.mg.gov.br/portal/acervo//covid19/decretos/5351\%20Republicado\%20por\%20in correcao.pdf. Acesso em: 22 nov. 2021.

UBERABA. Decreto $n^{\circ}$ 5372, de 20 de março de 2020. Determina o fechamento dos estabelecimentos que menciona, no âmbito do Município de Uberaba, Estado de Minas Gerais, decorrente do Coronavírus - COVID-19 e dá outras providências. Disponível em: http://www.uberaba.mg.gov.br/portal/acervo//covid19/decretos/5372..pdf. Acesso em: 22 nov. 2021.

UBERABA. Decreto $n^{\circ} 5555$, de 25 de maio de 2020. Prorroga a vigência do Decreto $N^{\circ}$ 5349/2020, que "Cria Grupo Estratégico de Gestão de Risco e Comitê Técnico-Científico para ações relacionadas ao Coronavírus, no âmbito do Município de Uberaba, Estado de Minas Gerais" 
e dá outras providências. Disponível em:

http://www.uberaba.mg.gov.br/portal/acervo//covid19/decretos/DECRETO\%20N\%205555,\%20DE \%2025\%20DE\%20MAIO\%20DE\%202020\%20-

\%20REPUBLICADO\%20POR\%20APERFEICOAMENTO\%20III.pdf. Acesso em: 22 nov. 2021.

UBERABA. Decreto $n^{\circ}$ 5836, de 31 de junho de 2020. Altera e Prorroga a vigência do Decreto $N^{\circ}$ 5379/2020, que "Cria, no âmbito do Município de Uberaba, Estado de Minas Gerais, Grupo de Trabalho para atendimento emergencial das pessoas e famílias em situação de vulnerabilidade social, econômica e financeira, decorrente da pandemia do Coronavírus - COVID-19" e dá outras providências. Disponível em:

http://www.uberaba.mg.gov.br/portal/acervo//covid19/decretos/DECRETO\%20N.\%205836,\%20DE \%2031\%20DE\%20JULHO\%20DE\%202020.pdf. Acesso em: 22 nov. 2021.

UBERABA CONTRA COVID. 2020. Disponível em:

http://uberabacontracovid.com.br/portal/conteudo,49164. Acesso em: 31 maio 2021.

VIANA, Humberto Lopes. Método para dimensionamento da quantidade ótima de sondas de produção em um campo de Petróleo: estudo de caso. Orientador: João Carlos Souza. 2003. 157 f. Dissertação (Mestrado em Engenharia de Produção) - Programa de Pós-Graduação em Engenharia de Produção, Universidade Federal de Santa Catarina, Florianópolis, 2003. Disponível em: http://repositorio.ufsc.br/xmlui/handle/123456789/86208. Acesso em: 20 nov. 2021.

WHO. Word Health Organization. WHO Director-General's opening remarks at the media briefing on COVID-19 - 11 March 2020. 2020. Disponível em:

https://www.who.int/dg/speeches/detail/who-director-general-s-opening-remarks-at-the-mediabriefing-on-covid-19---11-march-2020. Acesso em: 3 ago. 2021.

YADUVANSHI, D.; SHARMA, A.; MORE, P. V. Application of Queuing Theory to Optimize WaitingTime in Hospital Operations. Operations and Supply Chain Management, Surabaya, Indonesia, v. 12, n. 3, p. 165-174, 2019. DOI: http://doi.org/10.31387/oscm0380240. 